\title{
Multiple twinning and pseudosymmetry of Z-DNA hexamer duplexes
}

Zhipu Luo ${ }^{1}$, Miroslawa Dauter ${ }^{2}$, Zbigniew Dauter ${ }^{1}$

${ }^{1}$ National Cancer Institute, MCL, Argonne National Laboratory, Argonne, USA

${ }^{2}$ Leidos Biomedical Research, Inc., Basic Science Program, Argonne, USA.

The "classic" Z-DNA hexamer duplex d(CGCGCG)2 molecule is cylindrical, with diameter of $17 \AA$ and length of $22 \AA$, and has a tendency to crystallize in various pseudohexagonal forms. The PDB contains about 30 crystal structures of this duplex. Most of them are in the $P 2{ }_{1} 2{ }_{1} 2_{1}$ space group, but some in other symmetries. Those in $P 3_{2}, P 6_{5}$ or $P 6_{5} 22$ are approximate, since these groups assume continuous chains of DNA along the left-handed screw axes. We have obtained high resolution data for the two forms of $\mathrm{d}(\mathrm{CGCGCG})_{2}$, crystallized with either the $\mathrm{Mg}^{2+}$ or $\mathrm{Ca}^{2+}$ ions, and forming a sixfold pseudomerohedrally twinned crystals in the $P 2_{1}$ space group with either three or four symmetrically independent duplexes, respectively (the larger form is isomorphous with the previously described crystal of a DNA-RNA chimera). In both structures the gap (a missing phosphate moiety) is clearly supporting the symmetry interpretation of these crystals. The electron density map allowed to interpret detailed hydration patterns of the DNA and metal ions. 